\title{
Hemophagocytic lymphohistiocytosis in adults: collaborative analysis of 137 cases of a nationwide German registry
}

\author{
Sebastian Birndt ${ }^{1}$ (D) . Thomas Schenk ${ }^{1} \cdot$ Babett Heinevetter ${ }^{1} \cdot$ Frank M. Brunkhorst ${ }^{2} \cdot$ Georg Maschmeyer $^{3}$. \\ Frank Rothmann ${ }^{3} \cdot$ Thomas Weber $^{4} \cdot$ Markus Müller $^{5}$. Jens Panse ${ }^{6}$. Olaf Penack ${ }^{7} \cdot$ Roland Schroers $^{8} \cdot$ Jan Braess $^{9}$. \\ Norbert Frickhofen ${ }^{10}$. Stephan Ehl ${ }^{11}$. Gritta Janka ${ }^{12} \cdot$ Kai Lehmberg $^{12} \cdot$ Mathias W. Pletz $^{13} \cdot$ Andreas Hochhaus $^{1}$. \\ Thomas Ernst ${ }^{1} \cdot$ Paul La Rosée ${ }^{14}$
}

Received: 19 November 2019 / Accepted: 29 January 2020 / Published online: 20 February 2020

(C) The Author(s) 2020, corrected publication 2021

\begin{abstract}
Purpose Hemophagocytic lymphohistiocytosis (HLH) is a severe hyperinflammatory syndrome emerging from a deregulated immune response due to various triggers. In adults, systematic data are sparse, which is why recommendations on diagnosis and management have been adopted from pediatric guidelines. A nationwide clinical registry with associated consulting service as collaborative initiative of HLH-specialized pediatricians and hematologists was initiated to better characterize HLH in adults. Methods Patients with proven or suspected HLH were registered by 44 institutions. Both HLH-2004 diagnostic criteria and the HScore (www.saintantoine.aphp.fr/score/) were used to confirm HLH diagnosis. Data referring to underlying disease, treatment, outcome, clinical presentation and laboratory findings were recorded.

Results The study included 137 patients and provides the first systematic data on adult HLH in Germany. Median age was 50 years with a wide range (17-87 years), 87 patients (63.5\%) were male. Most common triggering diseases were infections in 61 patients (44.5\%) and malignancies in 48 patients (35\%). Virtually all patients had elevated ferritin concentrations, and $74 \%$ had peak concentrations greater than 10,000 $\mu \mathrm{g} / \mathrm{l}$. At time of analysis, 67 of 131 patients $(51 \%)$ had died. Patients with malignancy-associated HLH had the shortest median survival (160 days), however no statistically significant difference between subgroups was observed $(p=0.077)$. Platelets under $20^{*} 10^{9} / 1$ and low albumin concentrations $(<20 \mathrm{~g} / \mathrm{l})$ were associated with poor overall and 30-day survival.

Conclusion Close multidisciplinary case consultation and cooperation is mandatory when treating adult HLH patients. Early contact with reference centers is recommended, especially in relapsing or refractory disease.
\end{abstract}

Keywords HLH $\cdot$ Hemophagocytic lymphohistiocytosis $\cdot$ Sepsis $\cdot$ Inflammation $\cdot$ Cytokine storm

\section{Introduction}

Hemophagocytic lymphohistiocytosis (HLH) is a hyperinflammatory syndrome driven by excessive activation and stimulation of cytotoxic T-lymphocytes, natural killer T-cells and macrophages with subsequent cytokine storm and organ damage (Janka and Lehmberg 2014). In adults, this often fatal aberrant immune response most frequently is triggered by infections and malignancies, or a combination of these. Patients with long-term immunosuppression are at increased risk to develop HLH (Ramos-Casals et al.

Paul La Rosée

paul.larosee@sbk-vs.de

Extended author information available on the last page of the article
2014), as are patients with autoimmune/autoinflammatory disorders. By convention, and with impact on differential treatment, HLH in patients with autoimmune/autoinflammatory diseases is also called macrophage activation syndrome (MAS-HLH) (Emile et al. 2016). The wide spectrum of HLH-initiating conditions in adults is reflected by the term "acquired" or "secondary" HLH.

In contrast, primary HLH typically manifests in childhood, often has a family history, and is linked to mutations in genes involved in lymphocyte cytotoxicity. This includes PRF1, coding for perforin, or genes involved in the transport or exocytosis of perforin-containing lytic granules (Sepulveda and de Saint Basile 2017). Immunodeficiency syndromes, commonly associated with albinism, also predispose to HLH (Henkes et al. 2015). Clinically, a triad consisting 
of prolonged fever, hepatosplenomegaly and pancytopenia is common. However, a number of endogenous (i.e., genetic predisposition, preexisting inflammation) and exogenous factors (i.e., immunosuppression, triggering disease) play a role in HLH pathogenesis (Brisse et al. 2016). The spectrum of possible underlying conditions and patient characteristics is reflected by distinct clinical presentations which also can mimic other diseases, making timely diagnosis challenging. Specifically, HLH often is indistinguishable from sepsis or autoinflammatory diseases (e.g., adult-onset Still disease). As a result, and despite a high index of suspicion, there is high likelihood for mis- or underdiagnosing HLH, especially in intensive care units (Lachmann et al. 2018). HLH diagnosis is based on the HLH-2004 diagnostic criteria established by the Histiocyte Society (summarized in Table 1) (Henter et al. 2007). Of note, these criteria were established in the pediatric setting along the HLH-1994 and HLH-2004 trial protocols including patients up to 18 years (Bergsten et al. 2017; Trottestam et al. 2011). According to HLH-2004 study criteria, HLH can be diagnosed in a patient with at least 5 of 8 diagnostic criteria and/or disease-causing mutations in HLH-related genes. Recently, adaptation of diagnostic criteria has been proposed by French investigators, considering the impact of graded parameters and of state of immune competence on diagnostic accuracy (Fardet et al. 2014). The diagnostic algorithm is available as web-based tool to calculate HLH probability (https://saintantoine.aphp.fr/score/).

Since HLH in adults is a rare and most probably underreported syndrome, our current knowledge relies on case reports and series (Hayden et al. 2016; Ramos-Casals et al. 2014). Therefore, a clinical registry was initiated, aiming to better characterize and understand the spectrum of triggering conditions and management of adult HLH in Germany. Screening and inclusion of patients was based on a clinical consulting service that was made public by the Onkopedia platform HLHguideline (www.onkopedia.com) and via www.hlh-registry.org.

In this report, the first analysis of the registry including 137 patients $\geq 17$ years is presented. A special focus was put on underlying diseases, clinical and laboratory findings, and potential prognostic factors.

\section{Patients and Methods}

This registry for adult hemophagocytic lymphohistiocytosis was launched in August 2010, with the aim to collect data on epidemiology, treatment, clinical and laboratory characteristics, and outcome of affected patients. Data collection was based on a clinical consulting service for adult patients with suspected or proven HLH. At the time of analysis (June 30th 2017), 156 patients with suspected or proven HLH had been enrolled by 44 medical institutions. Patients with confirmed HLH were reported primarily from hematology/ oncology centers (38/44 centers). After informed consent, an online documentation form (available at www.hlh-regis try.org) was used for initial data submission. In addition, medical records were reviewed to obtain further information on clinical course and treatment. Anonymized patient data were subsequently recorded in an online-based OpenClinica database (Waltham, MA, US).

To confirm HLH diagnosis, patients were evaluated using both the HLH-2004 criteria (Table 1) and the HScore (supplementary Table 1, online available at: https://saintantoine.aphp. fr/score/) to quantify the probability of having HLH (Fardet et al. 2014). Of a total of 156 enrolled patients, 129 patients (82.7\%) were eligible for analysis on the basis of HLH-2004
Table 1 HLH-2004 diagnostic criteria according to (Henter et al. 2007)
At least one of either (1) or (2) must be fulfilled:

(1) Molecular diagnosis consistent with HLH

(2) At least 5 of the 8 following criteria:

a. Fever

b. Cytopenia of two or more lineages

Hemoglobin $<90 \mathrm{~g} / \mathrm{l}$, ANC $<1 \times 10^{9} / 1$, Plate-

lets $<100 \times 10^{9} / 1$

c. Splenomegaly

d. Hypertriglyceridemia and/or hypofibrinogenemia

e. Hyperferritinemia

f. Elevated sIL-2R (sCD25)

g. Low or absent NK-cell activity

h. Hemophagocytosis in bone marrow, spleen, or lymph node
Fasting triglycerides $\geq 3 \mathrm{mmol} / \mathrm{l}$ Fibrinogen $<1.5 \mathrm{~g} / 1$

Ferritin $\geq 500 \mu \mathrm{g} / \mathrm{l}$ sIL-2R $\geq 2400 \mathrm{U} / \mathrm{ml}$

$A N C$ absolute neutrophil count, $s I L-2 R$ soluble interleukin-2 receptor, $N K$-cell natural killer cell 
diagnostic criteria (i.e., molecular diagnosis and/or at least five out of eight HLH criteria). Furthermore, we included eight additional patients who met four diagnostic criteria and reached HLH-probability of more than $90 \%$ according to the HScore. Thus, 137 of 156 patients (88\%) were suitable for further analysis. A flow chart illustrating our approach is provided in the supplement (supplementary Fig. 1 ). According to the most likely triggering disease based on medical information, patients were categorized into four different subgroups for either malignancy-associated HLH, infection-associated HLH, MAS-HLH, or HLH due to an unknown trigger. In 20 patients with available blood or bone marrow samples, targeted perforin sequencing was performed.

\section{Statistical analysis}

Results are presented as median plus range, frequencies, or percentages. Overall survival was defined as time from date of diagnosis to date of death from any cause or date of last follow-up, respectively. Patients without available follow-up data were excluded from further analysis. Kaplan-Meier method was used to visualize median survival times, and the log-rank test was used to compare subgroups. Cox's proportional hazards model was used for univariate and multivariate analyses. Variables with a $p$ value $<0.05$ in univariate analysis were included in multivariate analysis to determine independent predictive factors. A backward stepwise selection procedure (Wald) was performed, with significance level for exclusion set at 0.1 . All statistical tests were two-sided. $p$ values $<0.05$ were considered statistically significant. Correlation between HLH-2004 diagnostic criteria and the HScore was analyzed using Pearson's $r$. All statistical analyses were performed using IBM SPSS Version 24 (IBM Corp., Armonk, N.Y., US).

\section{Genetic analysis}

Genomic DNA was isolated from peripheral blood samples or bone marrow according to manufacturer instructions using the QIAamp DNA Mini Kit (Qiagen, Hilden, Germany). Coding exons of the perforin gene (PRF1) were amplified by polymerase chain reaction (PCR). Sanger sequencing was performed using the ABI 3500 Genetic Analyzer (Thermo Fisher Scientific Inc., Waltham, MA, US), mutation analysis was done using Mutation Surveyor software (SoftGenetics LLC., State College, PA, US).

\section{Results}

\section{Patient characteristics}

Of 137 eligible patients, 50 patients were female (36.5\%) and 87 patients were male $(63.5 \%)$. Median age at diagnosis was 50 years, ranging from 17 to 87 years. Information on time to diagnosis from date of first symptoms was available for 124 patients, with a median of 10 days (range 1-93 days). In 27 patients (21.8\%), HLH diagnosis was made later than 21 days after first presentation. At onset of HLH, preceding immunosuppression (i.e., azathioprine treatment, cyclosporine in patients after allogeneic stem cell transplantation) was present in 58 of 128 patients $(45.3 \%)$. For all patients enrolled $(n=156)$, the HScore was calculated. There was a significant direct correlation between the HLH-2004 diagnostic criteria and the HScore $(R=0.75, p<0.001)$, a scatter diagram is provided in Supplementary Fig. 2. One hundred and twenty-two of 137 patients (89\%) eligible for further analysis had HScore values greater than 203 points, i.e., a probability for HLH of more than $90 \%$.

\section{Triggering diseases}

The most frequent triggering diseases in our cohort were infections ( $n=61,44.5 \%$ of all patients) and malignancies $(n=48,35 \%)$. In malignancy-associated HLH, hematologic neoplasia, in particular lymphomas of B-lymphoid origin represented the main cause. Myeloid disorders, e.g., acute myeloid leukemia, were seen in a minority $(n=7$, $5.1 \%)$. Active Herpes virus infections (i.e., evidence of viremia by PCR) such as EBV $(n=21,15.3 \%)$ or CMV $(n=4,2.9 \%)$ were most prevalent in infection-associated HLH. Six patients developed HLH due to viral infections after allogeneic stem cell transplantation (EBV, $n=5$; CMV, $n=1)$. HIV infection was identified in 4 patients, of whom two had HIV/HHV8 co-infection. Bacterial infections were diagnosed in five patients (3.6\%), while fungal infection was diagnosed in one patient. Visceral leishmaniasis was detected in three patients, of whom two had preceding immune-modulating treatments with adalimumab and tocilizumab, respectively. In 13 patients $(9.5 \%)$ an infection was the likely triggering disease, as these patients presented with elevated procalcitonin and showed evidence for an infectious source (i.e., radiological evidence, infiltrates in radiography or CT scan), however no infectious agent was identified in this group. HLH due to autoimmune/autoinflammatory diseases (MAS-HLH) was diagnosed in 13 patients (9.5\%), with adult-onset Still disease being predominant. In five patients, diseasecausing mutations in HLH-related genes were identified. In 15 patients (10.9\%), a trigger could not be identified. A detailed overview demonstrating the spectrum of etiologies is provided in Table 2 . 


\section{Clinical presentation and laboratory findings}

Clinical presentation in adult HLH included a variable combination of symptoms, though fever and splenomegaly were most common (in $98 \%$ and $86 \%$ of all patients, Table 3 ). 82 of 133 patients (62\%) fulfilled the clinical triad consisting of fever, splenomegaly and cytopenia of at least two lineages. Other common clinical findings included hepatomegaly (61\% of patients), renal failure (47\%), pulmonary symptoms such as respiratory insufficiency (33\%), and neurological symptoms (31\%). Based on laboratory parameters such as albumin, bilirubin, and transaminases, virtually all patients had liver dysfunction or damage, while 32 patients presented with bleeding complications or disseminated intravascular coagulation (DIC). Laboratory characteristics are presented in Table $4.73 \%$ of the patients had cytopenia of at least two hematopoietic lineages. Nearly all patients (99\%) had increased serum ferritin concentrations, with a median peak concentration of $30,281 \mu \mathrm{g} / \mathrm{l}$. One-hundred of 135 patients (74\%) showed peak values above 10,000 $\mu \mathrm{g} / \mathrm{l}$. Extreme ferritin concentrations $(>50,000 \mu \mathrm{g} / \mathrm{l})$ were found in 42 patients (31\%). Elevated concentrations of fasting triglycerides and soluble interleukin-2 receptor (sIL-2R) were documented in 70 and $94 \%$ of patients, respectively. Decreased fibrinogen concentrations were present in $41 \%$ of the patients. Hemophagocytosis in either bone marrow samples $(n=79)$ or lymph nodes $(n=2)$ was detected in 81 of 129 patients $(63 \%)$.

Functional immune response tests were performed in a proportion of patients. NK-cell degranulation assays were carried out in 21 patients, with pathologic results in 9 (43\%). Testing for signaling-lymphocytic-activation-molecule-associated protein (SAP) deficiency was done in 9 patients, a pathologic result was detected in one patient who was diagnosed with X-linked lymphoproliferative disease 1 (XLP1). Reduced perforin expression was found in three of nine patients who underwent testing (33\%), and subsequent genetic analysis in these patients revealed one homozygous (A91V) and one compound-heterozygous mutation (A91V/ $\mathrm{Q} 405 \mathrm{X})$ in the perforin gene; in the third patient with reduced perforin expression a heterozygous perforin mutation (A91V) was found. In two more patients, genetic causes were unraveled: Griscelli syndrome type 2 and X-linked lymphoproliferative disease 2 (XLP-2), respectively. Patient characteristics are summarized in Supplementary Table 2.

In addition, targeted sequencing of the perforin gene was performed using blood or bone marrow samples from a total of 20 patients, revealing two patients $(10 \%)$ carrying heterozygous A91V perforin mutations.
Table 2 Underlying conditions

\begin{tabular}{|c|c|}
\hline & $n(\%)$ \\
\hline Malignant diseases & $48(35.0)$ \\
\hline Myeloid & $7(5.1)$ \\
\hline AML & $3(2.2)$ \\
\hline CML & $1(0.7)$ \\
\hline MDS/MPN overlap syndrome & $3(2.2)$ \\
\hline B-Lymphoid & $30(21.9)$ \\
\hline DLBCL & $11(8.0)$ \\
\hline Intravascular large B-cell lymphoma & $1(0.7)$ \\
\hline Hodgkin lymphoma & $7(5.1)$ \\
\hline Mantle cell lymphoma & $1(0.7)$ \\
\hline Marginal zone lymphoma & $2(1.5)$ \\
\hline B-CLL & $2(1.5)$ \\
\hline B-ALL & $1(0.7)$ \\
\hline B-cell lymphoma (no further information) & $5(3.6)$ \\
\hline T-lymphoid & $10(7.3)$ \\
\hline Peripheral T-cell lymphoma & $2(1.5)$ \\
\hline NK/T-cell lymphoma & $2(1.5)$ \\
\hline T-ALL & $1(0.7)$ \\
\hline NK-cell leukemia & $1(0.7)$ \\
\hline Angioimmunoblastic T-cell lymphoma & $2(1.5)$ \\
\hline Anaplastic large cell lymphoma & $1(0.7)$ \\
\hline Enteropathy-associated T-cell lymphoma & $1(0.7)$ \\
\hline Solid & $1(0.7)$ \\
\hline Testicular-mixed tumor & $1(0.7)$ \\
\hline Infections & $61(44.5)$ \\
\hline Viral & $39(28.5)$ \\
\hline EBV & $21(15.3)$ \\
\hline EBV after allogeneic stem cell transplantation & $5(3.6)$ \\
\hline CMV & $4(2.9)$ \\
\hline CMV after allogeneic stem cell transplantation & $1(0.7)$ \\
\hline CMV/EBV coinfection & $3(2.2)$ \\
\hline H1N1/EBV coinfection & $1(0.7)$ \\
\hline HHV6 & $1(0.7)$ \\
\hline HIV & $2(1.5)$ \\
\hline HHV8/HIV coinfection & $2(1.5)$ \\
\hline VZV & $1(0.7)$ \\
\hline HSV & $1(0.7)$ \\
\hline Influenza A & $1(0.7)$ \\
\hline Parvovirus B19 & $1(0.7)$ \\
\hline Hantavirus & $1(0.7)$ \\
\hline Bacterial & $5(3.6)$ \\
\hline Proteus spp. & $1(0.7)$ \\
\hline Klebsiella pneumoniae & $1(0.7)$ \\
\hline Salmonella typhii & $1(0.7)$ \\
\hline Pseudomonas aeruginosa & $(0.7)$ \\
\hline Staphylococcus Epidermidis & $1(0.7)$ \\
\hline Fungal & $1(0.7)$ \\
\hline Histoplasma capsulatum & $1(0.7)$ \\
\hline Parasite & $3(2.2)$ \\
\hline Leishmaniasis & $3(2.2)$ \\
\hline
\end{tabular}


Table 2 (continued)

\begin{tabular}{ll}
\hline & $n(\%)$ \\
\hline Infection without documented pathogen & $13(9.5)$ \\
Autoimmune/inflammatory diseases & $13(9.5)$ \\
Adult-onset still disease & $8(5.8)$ \\
Rheumatoid arthritis & $2(1.5)$ \\
Systemic lupus erythematosus & $1(0.7)$ \\
Crohn's disease & $1(0.7)$ \\
ANCA negative vasculitis & $1(0.7)$ \\
Idiopathic & $15(10.9)$ \\
Total & 137 \\
\hline
\end{tabular}

Of 14 patients with malignancy-associated HLH, 12 also had EBV replication, one patient had CMV replication, and one patient had EBV replication and HIV infection. Percentages may not add to 100 because of rounding

$A M L$ acute myeloid leukemia, $C M L$ chronic myeloid leukemia, MDS/ MPN myelodysplastic/myeloproliferative neoplasm, $D L B C L$ diffuse large B-cell lymphoma, $C L L$ chronic lymphocytic leukemia, $A L L$ acute lymphocytic leukemia, $E B V$ epstein-Barr virus, $C M V$ cytomegalovirus, $H H V$ human herpes virus, $H I V$ human immunodeficiency virus, $V Z V$ varicella zoster virus, $H S V$ herpes simplex virus, $A N C A$ anti-neutrophil cytoplasmic antibody

\section{Treatment}

Most patients received anti-inflammatory treatment using glucocorticosteroids (124 of 137 patients, $90.5 \%$ ), either as monotherapy, in combination with other immunosuppressive agents or as part of chemotherapy regimens such as CHOEP (cyclophosphamide, doxorubicin, vincristine, etoposide, and prednisone) in cases of malignancy-associated HLH. Etoposide was administered in 70 patients $(51.1 \%)$, intravenous polyvalent immunoglobulins were given in 63 patients (46.0\%), and cyclosporine was used in 28 patients (20.4\%). In four patients with refractory or relapsing HLH, allogeneic hematopoietic stem cell transplantation was carried out. Rituximab was not only used as part of B-cell lymphoma chemotherapy, but also as a treatment option in EBV infected patients. Of 37 patients, who had more than 2000 EBV copies/ml in whole blood samples (quantitative PCR), $22(59.5 \%)$ received treatment with rituximab. Alemtuzumab (anti-CD52 antibody) or tocilizumab (anti-interleukin-6-receptor antibody) were used in three and two patients, respectively; anakinra, an interleukin-1-receptor antagonist, was applied in selected patients $(n=7)$. In three patients with refractory HLH, cytokine adsorption via column filtration (Cytosorb ${ }^{\circledR}$ ) was successfully deployed as salvage therapy.

\section{Outcome and prognostic factors}

In 131 patients follow-up data for survival analysis were available, while six patients were lost to follow-up. Median follow-up time from diagnosis was 154 days, and median overall survival was 267 days. Patients with malignancyassociated HLH had the shortest median survival (160 days), followed by those with idiopathic HLH (248 days), infection-associated HLH (641 days), and MAS-HLH (not reached). However, there was no statistically significant difference between these subgroups ( $p=0.077$ using logrank test). Figure 1 presents a Kaplan-Meier plot, showing overall survival of different subgroups. Overall, 67 of 131 patients died (51.1\%); 27 patients (20.6\%) died within 30 days from date of HLH diagnosis. Multiple organ failure was the most common cause of death. To identify possible

Table 3 Clinical manifestations

\begin{tabular}{lll}
\hline & Number of patients & $(\%)$ \\
\hline Fever & $134 / 137$ & 98 \\
Splenomegaly & $115 / 133$ & 86 \\
Triad of fever, splenomegaly, cytopenia & $82 / 133$ & 62 \\
Cytopenia (at least two lineages)* & $99 / 135$ & 73 \\
Neutropenia (ANC $\left.<1 \times 10^{9} / 1\right)$ & $51 / 119$ & 43 \\
Anemia (Hemoglobin $<90$ g/l) & $97 / 135$ & 72 \\
Thrombocytopenia (Platelets $\left.<100 \times 10^{9} / 1\right)$ & $111 / 135$ & 82 \\
Hemophagocytosis (bone marrow, spleen, or lymph node) & $81 / 129$ & 63 \\
Hepatomegaly & $63 / 103$ & 61 \\
Renal involvement, acute renal failure & $52 / 111$ & 47 \\
Neurological symptoms & $41 / 131$ & 31 \\
Bleeding complications, manifest DIC & $44 / 136$ & 32 \\
Pulmonary involvement, respiratory insufficiency & $40 / 121$ & 33 \\
Peripheral lymphadenopathy & $38 / 116$ & 33 \\
\hline
\end{tabular}

ANC absolute neutrophil count, DIC disseminated intravascular coagulation

$*$ ANC $<1 \times 10^{9} / 1$; Hemoglobin $<90$ g/l; Platelets $<100 \times 10^{9} / 1$ 
Table 4 Laboratory findings. If not specifically marked, all data are presented using frequencies; corresponding percentages are presented in parentheses

\begin{tabular}{|c|c|c|c|c|c|}
\hline & Overall & M-HLH & I-HLH & MAS-HLH & Idiopathic \\
\hline Number of patients & 137 & 48 & 61 & 13 & 15 \\
\hline Median age in years (range) & $50(17-87)$ & $60(18-87)$ & $43(17-71)$ & $40(18-78)$ & $55(19-81)$ \\
\hline Male $(n)$ & 87 & 32 & 40 & 7 & 8 \\
\hline Female $(n)$ & 50 & 16 & 21 & 6 & 7 \\
\hline \multicolumn{6}{|l|}{ HLH 2004 criteria } \\
\hline Fever & $134 / 137(98)$ & $47 / 48(98)$ & $60 / 61(98)$ & $13 / 13(100)$ & $14 / 15(93)$ \\
\hline Splenomegaly & $115 / 133 *(86)$ & $42 / 45(93)$ & $48 / 60(80)$ & $12 / 13(92)$ & $13 / 15(87)$ \\
\hline Cytopenias (of at least two lineages) & $99 / 135(73)$ & $42 / 48(88)$ & $44 / 59(75)$ & $5 / 13(38)$ & $8 / 15(53)$ \\
\hline Neutropenia $\left(\right.$ ANC $\left.<1 \times 10^{9} / 1\right)$ & $51 / 119(43)$ & $18 / 42(43)$ & $23 / 50(46)$ & $3 / 12(25)$ & $7 / 15(47)$ \\
\hline Anemia $($ Hemoglobin $<90 \mathrm{~g} / \mathrm{l})$ & $97 / 135(72)$ & $39 / 48(81)$ & $40 / 59(68)$ & $8 / 13(62)$ & $10 / 15(67)$ \\
\hline Thrombocytopenia (Platelets $\left.<100 \times 10^{9} / 1\right)$ & $111 / 135(82)$ & $45 / 48(94)$ & $52 / 59(88)$ & $6 / 13(46)$ & $8 / 15(53)$ \\
\hline Hypertriglyceridemia (>3 mmol/l) & $86 / 123(70)$ & $36 / 44(82)$ & $35 / 54(65)$ & $8 / 12(67)$ & $7 / 13(54)$ \\
\hline Hypofibrinogenemia $(<1.5 \mathrm{~g} / \mathrm{l})$ & $52 / 127(41)$ & $20 / 44(45)$ & $23 / 56(41)$ & $3 / 12(25)$ & $6 / 15(40)$ \\
\hline Ferritin elevation $(>500 \mu \mathrm{g} / \mathrm{l})$ & $134 / 135(99)$ & $47 / 48(98)$ & $59 / 59(100)$ & $13 / 13(100)$ & $15 / 15(100)$ \\
\hline Ferritin peak $>10,000 \mu \mathrm{g} / 1$ & $100 / 135(74)$ & $37 / 48(77)$ & $45 / 59(76)$ & $9 / 13(69)$ & $9 / 15(60)$ \\
\hline Ferritin peak $>50,000 \mu \mathrm{g} / 1$ & $42 / 135(31)$ & $11 / 48(23)$ & $24 / 59(41)$ & $4 / 13(31)$ & $3 / 15(20)$ \\
\hline Ferritin at initial presentation, Median $(\mu \mathrm{g} / \mathrm{l})$ range & $\begin{array}{l}6,747 \\
479-143,210\end{array}$ & $\begin{array}{l}6,696 \\
479-70,100\end{array}$ & $\begin{array}{l}11,782 \\
563-143,210\end{array}$ & $\begin{array}{l}4,175 \\
1,431-15,733\end{array}$ & $\begin{array}{l}6,373 \\
2,175-50,000\end{array}$ \\
\hline Ferritin peak values, Median $(\mu \mathrm{g} / \mathrm{l})$ range & $\begin{array}{l}30,281 \\
479-2,632,220\end{array}$ & $\begin{array}{l}24,404 \\
479-526,259\end{array}$ & $\begin{array}{l}39,504 \\
1,254-2,632,220\end{array}$ & $\begin{array}{l}30,554 \\
1,243-186,833\end{array}$ & $\begin{array}{l}16,146 \\
3,855-188,620\end{array}$ \\
\hline Soluble CD25 (sIL-2R) $(>2,400 \mathrm{U} / \mathrm{ml})$ & $103 / 109(94)$ & $40 / 41(98)$ & $46 / 47(98)$ & $7 / 11(64)$ & $10 / 10(100)$ \\
\hline Median $(\mathrm{U} / \mathrm{ml})$ range & $\begin{array}{l}7,500 \\
1,194-108,640\end{array}$ & $\begin{array}{l}11,298 \\
1,194-108,640\end{array}$ & $\begin{array}{l}7,500 \\
2,125-70,300\end{array}$ & $\begin{array}{l}5,080 \\
1,333-26,660\end{array}$ & $\begin{array}{l}7,015 \\
2,472-30,000\end{array}$ \\
\hline Low or absent NK-cell activity & $9 / 21(43)$ & $2 / 2(100)$ & $6 / 16(38)$ & $0 / 2(0)$ & $1 / 1(100)$ \\
\hline Low NK-cell count (FACS) & $29 / 44(66)$ & $7 / 11(64)$ & $18 / 26(69)$ & $2 / 4(50)$ & $2 / 3(67)$ \\
\hline Hemophagocytosis $^{+}$ & $81 / 129(63)$ & $24 / 44(55)$ & $35 / 57(61)$ & $8 / 13(62)$ & $14 / 15(93)$ \\
\hline \multicolumn{6}{|l|}{ Other } \\
\hline Elevated alanine aminotransferase (ALAT) & $111 / 131(85)$ & $38 / 48(79)$ & $50 / 56(89)$ & $11 / 13(85)$ & $12 / 14(86)$ \\
\hline Elevated ALAT $>2.5 \times$ ULN & $75 / 131(57)$ & $20 / 48(42)$ & $39 / 56(70)$ & $8 / 13(62)$ & $8 / 14(57)$ \\
\hline Elevated aspartate aminotransferase (ASAT) & $123 / 133(92)$ & $43 / 47(91)$ & $54 / 58(93)$ & $12 / 13(92)$ & $14 / 15(93)$ \\
\hline Elevated ASAT $>2.5 \times \mathrm{ULN}$ & 98/133 (74) & $32 / 47(68)$ & $47 / 58(81)$ & $8 / 13(62)$ & $11 / 15(73)$ \\
\hline Elevated total bilirubin level & $90 / 132(68)$ & $33 / 48(69)$ & $45 / 57(79)$ & $5 / 13(38)$ & $7 / 14(50)$ \\
\hline Elevated total bilirubin level $>2.5 \times \mathrm{ULN}$ & $59 / 132(45)$ & $22 / 48(46)$ & $30 / 57(53)$ & $3 / 13(23)$ & 4/14 (29) \\
\hline Hypoalbuminemia $(<35 \mathrm{~g} / \mathrm{l})$ & 109/111 (98) & $39 / 40(98)$ & $48 / 49(98)$ & $8 / 8(100)$ & $14 / 14(100)$ \\
\hline Albumin $<20 \mathrm{~g} / \mathrm{l}$ & $39 / 111(35)$ & $15 / 40(38)$ & $19 / 49(39)$ & $3 / 8(38)$ & 2/14 (14) \\
\hline Elevated lactate dehydrogenase (LDH) & $126 / 133(95)$ & $45 / 47(96)$ & $54 / 58(93)$ & $13 / 13(100)$ & $14 / 15(93)$ \\
\hline Elevated $\mathrm{LDH}>2.5 \times \mathrm{ULN}$ & $97 / 133(73)$ & $31 / 47(66)$ & $46 / 58(79)$ & $8 / 13(62)$ & $12 / 15(80)$ \\
\hline Elevated C-reactive protein level & $129 / 132(98)$ & $46 / 48(96)$ & $56 / 56(100)$ & $13 / 13(100)$ & $14 / 15(93)$ \\
\hline
\end{tabular}

M-HLH malignancy-associated HLH, I-HLH infection-associated HLH, MAS-HLH macrophage activation syndrome, ANC absolute neutrophil count, $s I L-2 R$ soluble interleukin-2 receptor, $N K$-cell natural killer cell, FACS Fluorescence-activated cell sorting, $U L N$ upper limit of normal

*One patient had splenectomy prior to HLH diagnosis

${ }^{+}$Assessed morphologically in either bone marrow, spleen, or lymph node

prognostic factors for both overall and 30-day mortality, univariate and multivariate analyses were conducted. In univariate analysis, age over 50 years, low absolute neutrophil count, hemoglobin below $8.2 \mathrm{~g} / \mathrm{dl}$, platelet count below $20 * 10^{9} / 1$, ferritin concentration above $10,000 \mu \mathrm{g} / \mathrm{l}$, albumin concentration below $20 \mathrm{~g} / \mathrm{l}$, and more than fivefold increased bilirubin concentration were associated with a poor outcome (Table 5). By multivariate analysis, age over 50 years (HR 1.811; 95\% CI 1.020-3.217; $p=0.043$ ), absolute neutrophil count below $1 * 10^{9} / 1$ (HR $1.861 ; 95 \%$ CI 1.056-3.281; $p=0.032$ ), platelet count below $20 * 10^{9} / 1$ (HR 2.243; 95\% CI 1.210-4.158; $p=0.010$ ) and albumin 
Fig. 1 Kaplan-Meier plot showing overall survival for different HLH subgroups. Patients with malignancy-associated HLH had the shortest median survival time, although no statistically significant difference between the subgroups was observed (log-rank test: $p=0.077$ )

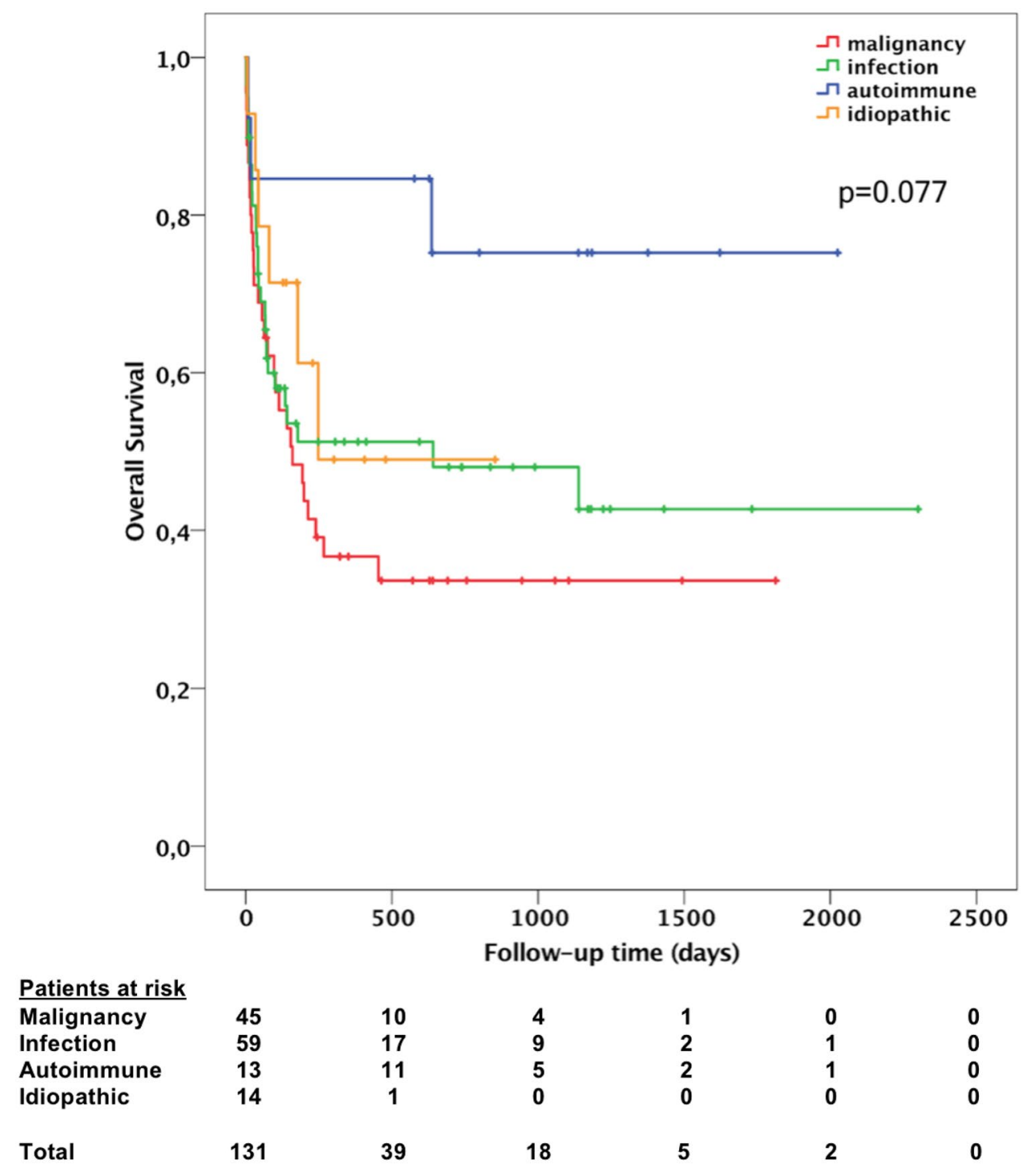

Table 5 Univariate analysis of possible predictors of mortality

\begin{tabular}{|c|c|c|c|c|c|c|}
\hline \multirow[t]{2}{*}{ Prognostic factor } & \multicolumn{3}{|c|}{ Overall } & \multicolumn{3}{|c|}{ Death within 30 days } \\
\hline & HR & $95 \% \mathrm{CI}$ & Significance & HR & $95 \% \mathrm{CI}$ & Significance \\
\hline Age $>50$ years & 1.994 & $1.214-3.276$ & $0.006^{*}$ & 1.838 & $0.841-4.014$ & 0.127 \\
\hline Gender male vs. female & 1.032 & $0.628-1.695$ & 0.902 & 0.737 & $0.345-1.574$ & 0.430 \\
\hline Neutrophils $<1 * 10^{9} / 1$ & 1.970 & $1.162-3.341$ & $0.012 *$ & 2.615 & $1.108-6.171$ & $0.028 *$ \\
\hline Hemoglobin $<8.2 \mathrm{~g} / \mathrm{dl}$ & 1.750 & $1.070-2.862$ & $0.026^{*}$ & 1.471 & $0.682-3.170$ & 0.325 \\
\hline Platelets $<20^{*} 10^{9} / 1$ & 2.133 & $1.270-3.583$ & $0.004 *$ & 3.646 & $1.711-7.768$ & $0.001 *$ \\
\hline Fibrinogen $\leq 1.5 \mathrm{~g} / \mathrm{l}$ & 1.042 & $0.631-1.723$ & 0.872 & 0.633 & $0.273-1.466$ & 0.286 \\
\hline Ferritin $>10,000 \mu \mathrm{g} / 1$ & 2.025 & $1.034-3.965$ & $0.040 *$ & 1.216 & $0.491-3.012$ & 0.673 \\
\hline Presence of hemophagocytosis & 0.674 & $0.407-1.116$ & 0.125 & 0.654 & $0.298-1.433$ & 0.288 \\
\hline Albumin $<20 \mathrm{~g} / \mathrm{l}$ & 2.318 & $1.386-3.877$ & $0.001 *$ & 2.868 & $1.273-6.461$ & $0.011^{*}$ \\
\hline More than fivefold increased Bilirubin & 2.272 & $1.395-3.700$ & $0.001 *$ & 1.576 & $0.741-3.352$ & 0.238 \\
\hline
\end{tabular}

$H R$ hazard ratio, $C I$ confidence interval

*Indicates statistically significant values 
concentration below $20 \mathrm{~g} / \mathrm{l}$ (HR 2.606; 95\% CI 1.490-4.561; $p=0.001$ ) were independent predictors of poor overall survival (Table 6). Repeating the analysis for death within 30 days after HLH diagnosis, low absolute neutrophil count, platelets below $20^{*} 10^{9} / 1$, and albumin below $20 \mathrm{~g} / \mathrm{l}$ were associated with early death in univariate analysis. Multivariate analysis revealed platelets below $20 * 10^{9} / 1$ (HR 3.446; 95\% CI 1.471-8.073; $p=0.004)$ and albumin below $20 \mathrm{~g} / 1$ (HR 2.531; 95\% CI $1.067-6.005 ; p=0.035$ ) to be independent predictors for early death within 30 days after HLH diagnosis.

\section{Discussion}

Hemophagocytic lymphohistiocytosis (HLH) constitutes a severe hyperinflammatory syndrome emerging from a deregulated immune system due to various triggering conditions. Despite being first described back in 1928 by Tschistowitsch and Bykowa as systemic reticulosis (presumably the first report ever) (Tschistowitsch and Bykowa 1928) and 1939 by Scott and Robb-Smith as histiocytic medullary reticulosis (first report in english) (Bodley Scott and Robb-Smith 1939), HLH in adult patients was neglected for a long time. Increasing awareness developed during the past decade resulting in a growing number of publications (Arca et al. 2015; Bachier Rodriguez and Ritchie 2016; Berliner et al. 2016; Delavigne et al. 2014; Fardet et al. 2010; Halacli et al. 2016; Li et al. 2014, 2015; Machaczka et al. 2011; Otrock and Eby 2015; Park et al. 2012; Ramos-Casals et al. 2014; Riviere et al. 2014; Schram et al. 2016; Tamamyan et al. 2016). However, only few case series on adult HLH are available and essential parts of current management standards for

Table 6 Multivariate analysis

\begin{tabular}{llll}
\hline & Hazard ratio & $95 \%$ CI & Significance \\
\hline $\begin{array}{llll}\text { Overall survival } \\
\text { Age }>50 \text { years }\end{array}$ & 1.811 & $1.020-3.217$ & $0.043^{*}$ \\
Neutrophils $<1 * 10^{9} / 1$ & 1.861 & $1.056-3.281$ & $0.032^{*}$ \\
Hemoglobin $<8.2 \mathrm{~g} / \mathrm{dl}$ & 1.133 & $0.614-2.090$ & 0.689 \\
Platelets $<20^{*} 10^{9} / 1$ & 2.243 & $1.210-4.158$ & $0.010^{*}$ \\
Ferritin $>10,000 \mu \mathrm{g} / 1$ & 1.381 & $0.645-2.960$ & 0.406 \\
Albumin $<20 \mathrm{~g} / 1$ & 2.606 & $1.490-4.561$ & $0.001^{*}$ \\
More than fivefold & 1.323 & $0.693-2.526$ & 0.396 \\
$\quad$ increased bilirubin & & & \\
Death within 30 days & & & \\
Neutrophils $<1 * 10^{9} / 1$ & 1.746 & $0.708-4.304$ & 0.226 \\
Platelets $<20 * 10^{9} / 1$ & 3.446 & $1.471-8.073$ & $0.004^{*}$ \\
Albumin $<20 \mathrm{~g} / 1$ & 2.531 & $1.067-6.005$ & $0.035^{*}$ \\
\hline
\end{tabular}

CI Confidence interval

*Indicates statistically significant values adult patients (i.e., diagnostic criteria, treatment protocols) are adapted from pediatric HLH guidelines. Therefore, a national registry including an associated consulting service was established to collect cases of adult HLH patients and contribute to a better understanding and improved clinical management of this rare and still often fatal syndrome. This registry is part of a campaign to sensitize clinicians in Germany for potential HLH.

HLH in adults emerges from various underlying conditions, as demonstrated in an overview by Ramos-Casals (Ramos-Casals et al. 2014). Considering the data of almost 2200 patients from published case series, infections and malignancies were the most common triggers, followed by autoimmune/autoinflammatory diseases. HLH can also arise during or after chemotherapy, or in the context of organ or stem cell transplantation (Delavigne et al. 2014; Lehmberg et al. 2015).

Of note, underlying conditions such as infections or malignancies alone can lead to the clinical picture of HLH if not adequately controlled; besides, infections may also act as trigger in patients with preexisting autoinflammatory or malignant disorders.

Most recently, reports of cytokine release syndromes due to cellular and bispecific T-cell engaging immunotherapies came into focus, as they share pathophysiologic aspects of HLH and present with a similar clinical phenotype (Lee et al. 2014; Teachey et al. 2013). In our cohort, the triggering entities were in line with the literature, with infections (44.5\%) and malignancies (35\%) being most frequent. In malignancy-triggered HLH, lymphoma, especially of B-lymphoid origin, was the predominant trigger. This is in contrast to Asian countries, where T-cell lymphoma, in accordance with their higher prevalence, are the most common lymphoma subtype in malignancy associated HLH (Han et al. 2007; La Rosee 2015a; Li et al. 2014).

In infection-associated HLH, viral infections, especially with herpes viruses such as EBV or CMV, were most common. In several patients, bacterial, fungal, or parasitic infections were identified (Table 2). Interestingly, in three patients visceral leishmaniasis was the triggering disease. Two of the affected patients were immunosuppressed by previous treatment with biologicals. In one of three patients, leishmaniasis was diagnosed by polymerase chain reaction (PCR), while bone marrow morphology was negative for intracellular Leishmania amastigotes. Therefore, including leishmania PCR in the diagnostic bone marrow workup is strongly recommended, to safely protect patients from harmful immunosuppression. Specific treatment using liposomal amphotericin is available (Bode et al. 2014). In our registry, one patient was refugee from Albania, and two patients were German tourists returning from Mallorca and Crete. For risk assessment of potential leishmania exposition, World Health Organization (WHO) maps of endemic distribution can be 
accessed via https://www.who.int. Collaboration with clinical infectiologists is mandatory.

Leishmania-associated HLH demonstrates how important it is to identify the underlying disease, and not to be satisfied with the diagnosis "HLH". Experience in a French pediatric HLH-series, where three children received etoposide with the diagnosis HLH of unknown origin, and the clinical course of one of our registry patients, who received etoposide after Leishmania was excluded by bone marrow morphology only (without PCR-testing), highlights the risk of treating HLH according to standard protocols without considering specific treatment of the triggering disease. This can also be challenging when lymphomas triggering HLH are masked by inflammatory infiltrates in tissue samples from lymph node, liver, spleen or skin, preventing the pathologist from detecting the malignant tissue component. It is therefore pivotal to carry on sequential diagnostic reassessment by imaging studies, laboratory tests and tissue biopsies along with ongoing treatment. In some cases, splenectomy has been shown to demask lymphomas despite non-informative imaging studies including PET-scan (Jing-Shi et al. 2015).

In general, HLH treatment is based upon three columns: (a) immunosuppression using dexamethasone \pm polyvalent immunoglobulins and-if needed-more aggressive immune cell depletion using etoposide, anti-thymocyte globulin or alemtuzumab to interrupt pathologic immune activation, (b) classic measures of intensive care medicine to sustain organ function and prevent severe bleeding, and (c) specific trigger-directed therapy, i.e., chemotherapy, antimicrobial agents or cytokine neutralization. Treatment strategies in adults encompass main components adapted from protocols developed for the control of primary HLH in children (i.e., HLH-94 and HLH-2004 protocol), but are tailored to the individual patient, depending on HLH severity and the underlying trigger (La Rosee 2015b). Upcoming strategies include more specific therapies [i.e., antibodies such as tocilizumab (Teachey et al. 2013), the interferon-gamma antibody emapalumab (Jordan et al. 2015), small molecules such as the Jak1/2-inhibitor ruxolitinib (La Rosee 2016; Sin and Zangardi 2017) or cytokine adsorption, which was successfully used in three of our patients. The latter treatment might be useful to bridge the time from cytokine-dependent organ failure, precluding cytotoxic treatment, to definitive causative treatment (Frimmel et al. 2014; Greil et al. 2017). The intensity of immunosuppression in the pediatric HLH1994 protocol is neither suitable nor required in the majority of adult HLH patients (Bergsten et al. 2017; Ehl et al. 2018). It seems rather advisable to avoid prolonged immunosuppression, in particular to reduce the risk of subsequent infections. Fever episodes after HLH-directed immunosuppression require careful evaluation for potential secondary infections to avoid harming HLH-salvage treatments.
Primary clinical presentation in our cohort was variable. Besides a triad consisting of fever, hepatosplenomegaly, and cytopenia ( $62 \%$ of our patients), patients presented with renal failure, liver dysfunction (i.e., elevated bilirubin, low albumin, coagulation disorder), neurological symptoms (impaired consciousness, seizures), or bleeding complications. Of note, histological or cytological proof of hemophagocytosis is not necessarily required for diagnosing HLH despite being the eponymous feature. In several studies hemophagocytosis was missing in up to $40 \%$ of the patients (Otrock and Eby 2015; Riviere et al. 2014). In our cohort, only $63 \%$ of the patients had hemophagocytosis according to assessment of the respective pathologist. On the other hand, hemophagocytosis may also appear in sepsis or rheumatologic disorders, reducing its specificity for HLH (Gupta et al. 2008). Perhaps more rigorous morphological criteria as proposed by Gars et al. might help to differentiate between HLH and other inflammatory conditions. In this study on 78 patients with or without HLH, HLH was strongly associated with hemophagocytosis of granulocytes, nucleated erythrocytes and at least one hemophagocyte engulfing multiple nucleated cells (Gars et al. 2018). Future studies should verify these findings and its feasibility in evaluation of potential HLH.

Diversity of clinical pictures in adult HLH often leads to delayed diagnosis and presumably a high number of unreported cases, as most of the aforementioned symptoms are found in a variety of other diseases (Lachmann et al. 2018). In the analysis of our data, median time to diagnosis was 10 days. However, in about $20 \%$ of the patients, time to diagnosis was longer than 3 weeks, highlighting the difficulties in recognizing HLH in due time. Red flags are persisting fever despite broad antibiotic therapy and cytopenia, or a sepsis-like clinical picture, without detected pathogen and only poor response to anti-infective treatment.

Highly elevated ferritin concentrations are a hallmark in HLH. In our study, virtually all patients had elevated ferritin (99\%), and $74 \%$ of the patients had peak concentrations above $10,000 \mu \mathrm{g} / \mathrm{l}$, while $31 \%$ even had a ferritin peak concentration above $50,000 \mu \mathrm{g} / \mathrm{l}$. These findings are in accordance with other studies suggesting that ferritin concentrations above $2000 \mu \mathrm{g} / \mathrm{l} \mathrm{might} \mathrm{be} \mathrm{more} \mathrm{specific}$ than the threshold used in the HLH-2004 criteria (Fardet et al. 2010; Otrock and Eby 2015; Parikh et al. 2014; Schram et al. 2016). Therefore, extremely high ferritin in the absence of a known iron metabolism disorder, hemolysis or multiple transfusions should lead to evaluation of possible HLH, despite limited specificity in adults (Allen et al. 2008; Otrock et al. 2017; Schram et al. 2015). Besides ferritin, soluble interleukin-2 receptor is a useful diagnostic and monitoring tool, which also might have prognostic significance (Hayden et al. 2017; Zhang et al. 2011b). A high ratio between sIL-2R and ferritin has been suggested as a 
potential marker for lymphoma-associated HLH and might help in diagnosing patients with yet undetected lymphoma (Lin et al. 2017; Tsuji et al. 2014). Based on the published literature and the experience from our registry we strongly recommend extensive search for possible lymphoma in HLH patients presenting with markedly elevated sIL-2R levels.

In this context, the HScore may be a valuable add-on tool for clinicians if HLH is suspected. The score was developed by a French working group in 2014 to assess the probability of having reactive hemophagocytic syndrome, and to distinguish HLH from other medical conditions such as sepsis (Fardet et al. 2014). Free online availability, graduation of laboratory parameters, and exclusion of elaborate tests, e.g., NK-cell activity, are main advantages of the score. In our cohort, we found a strong correlation between the HScore and HLH-2004 criteria $(r=0.75, p<0.001)$, and $89 \%$ of the patients had a probability of more than $90 \%$ for HLH according to the HScore (Supplementary Fig. 2). As its calculation is easy and only takes a few minutes, the use of the HScore in addition to HLH-2004 criteria is recommended when evaluating patients with suspected HLH.

NK cell activity was tested in a proportion of our patients. Yet, functional testing (i.e., degranulation or expression assays) may only be advisable in selected patients, i.e., in young male patients with EBV-associated HLH, HLHrelapse or in patients with suspected primary immunodeficiency (i.e., albinism) (Lehmberg and Ehl 2013). If pathologic results are found, a subsequent genetic analysis is suggested, not to miss cases with late-onset hereditary disease. Previous studies demonstrated that mutations in HLH-predisposing genes are present in up to $10 \%$ of adult patients with HLH (Wang et al. 2014; Zhang et al. 2011a). In our registry, five patients showed HLH-associated gene mutations-a number possibly underestimating the true value since only a proportion of patients was tested. Besides known disease-causing mutations, monoallelic mutations in HLH-related genes have been described in affected patients, however their impact on HLH development is not fully clear. In a report from the Italian HLH registry, Cetica et al. found monoallelic mutations in 43 of 426 patients analyzed (10.1\%), and postulated a potential influence on HLH pathogenesis (Cetica et al. 2016). Of note, the monoallelic perforin A91V mutation occurs in up to $9 \%$ of healthy individuals and, as the cumulative incidence of HLH is much lower, cannot be regarded as genetically causative for HLH development (Busiello et al. 2006; Zur Stadt et al. 2004).

Despite advances in treatment strategies, the prognosis in adult HLH is still poor. In previous analyses, HLH mortality rates up to $75 \%$ were reported (Parikh et al. 2014; Schram et al. 2016; Shabbir et al. 2011). In our cohort, the overall mortality rate was 51\% (67/131). According to various studies, patients with malignancy-associated HLH have the worst prognosis (Machaczka et al. 2011; Otrock and Eby 2015;
Parikh et al. 2014; Shabbir et al. 2011; Tamamyan et al. 2016). An overview provided by Daver et al. found a median survival time between 1.2 and 2.4 months for patients with malignancy-associated HLH (Daver et al. 2017). Accordingly, patients with underlying malignancy had the shortest median survival in our cohort, whereas patients with nonmalignancy HLH tended to have a better prognosis, although there was no statistical significance (Fig. 1).

Several adverse prognostic factors such as male sex, higher age, malignancy, low platelet counts, or low albumin have been described in adult HLH (Arca et al. 2015; Li et al. 2014; Oto et al. 2015; Otrock and Eby 2015; Parikh et al. 2014). In our study, age above 50 years, neutrophil count less than $1 * 10^{9} / 1$, platelets under $20 * 10^{9} / 1$, and albumin under $20 \mathrm{~g} / \mathrm{l}$ predicted a poor overall survival. These prognostic factors, together with underlying trigger and the extent of laboratory alterations, might help to classify HLH patients into different risk groups. This will allow tailoring HLH-directed and adjusting trigger-directed therapy, i.e., the early use of etoposide in severe HLH cases or consolidation with autologous stem cell transplantation in patients with lymphoma-associated HLH.

Altogether, our study includes a relatively large number of patients from 44 different institutions and thus currently provides the best overview on HLH in adults in Germany. The major limitation arises from retrospective data acquisition and analysis.

HLH in adults is clinically highly heterogeneous with patients presenting in virtually all subdisciplines depending on initial symptoms and etiology. With increasing age, lymphoma becomes the most prevalent HLH-trigger, yet histologic proof of lymphoma often is masked by HLHassociated lymphoproliferation. High suspicion and relentless search for the underlying disease together with interdisciplinary diagnostics and care are cornerstones for reversing the dismal prognosis of HLH. Further cooperative research is necessary to systematically study this rare syndrome and to define optimal treatment algorithms, including novel targeted therapies (i.e., biologic agents).

\section{Appendix: Participating centers}

Universitätsklinikum Aachen—J. Panse, B. Voss, D. Goy. Klinikum Augsburg-K. Hirschbühl.

Charité - Universitätsmedizin Berlin, Campus Virchow-Klinikum-O. Penack.

Vivantes Auguste-Viktoria-Klinikum Berlin-M. Müller, H. Stocker.

Evangelisches Klinikum Bethel, Bielefeld-F. Weißinger.

Medizinische Universitätsklinik Knappschaftskrankenhaus Bochum-R. Schroers. 
Klinikum Chemnitz-R. Herbst, A. Morgner.

Krankenhaus Dornbirn-W. Bair.

Klinikum Dortmund-M. Unnewehr.

HELIOS Klinikum Erfurt—H. Sayer.

Universitätsklinikum Erlangen-S. Krause, E. Hofmann.

Klinikum Esslingen-M. Geißler.

Klinikum Frankfurt Höchst-F. Scholten.

Universitätsklinikum Freiburg-S. Ehl, F. Röther.

Universitätsklinikum Gießen-F. Ohliger, J. Trauth.

Universitätsmedizin Göttingen-D. Burghardt.

Universitätsmedizin Greifswald-M. Lerch, C. Hirt.

Universitätsklinikum Halle (Saale)—T. Weber.

Universitätsklinikum Hamburg-Eppendorf-S. Schliffke,

Christina Dicke.

Klinikum Heidenheim-M. Müller.

Universitätsklinikum Schleswig-Holstein Kiel-M. Ritgen, D. Schewe.

Universitätsklinikum Köln—S. Krämer.

St. Marien- und St. Annastiftskrankenhaus Ludwigshafen-P. Meier.

Universitätsmedizin Mainz-P. Wölfinger.

Städtisches Klinikum München Schwabing-M. Starck.

Klinikum der Universität München, Standort Großhadern-M. Subklewe, V. Bücklein.

Rotkreuzklinikum München-M. Hentrich.

Gemeinschaftspraxis für Hämatologie und Onkologie Münster-R. Liersch.

Universitätsklinikum Münster-B. Baumgarten, A. Schulze, M. Mohr.

Klinikum Nürnberg Nord-S. Dressler.

Klinikum Ernst von Bergmann Potsdam-G.

Maschmeyer, F. Rothmann.

Krankenhaus Barmherzige Brüder Regensburg-J. Braess.

Universitätsklinikum Regensburg-T. Lange, M. Grube,

M. Fante.

Universitätsmedizin Rostock-D. Gläser, J. Lakner, M. Bärenklau.

Klinikum Sindelfingen-Böblingen-M. Ritter.

Universitätsklinikum Tübingen-R. Riessen, M. Haap.

Universitätsklinikum Ulm - A. Viardot.

Schwarzwald-Baar Klinikum Villingen-Schwenningen-

M. Henkes.

Klinikum Wels-Grieskirchen-S. Burgstaller.

HELIOS Dr. Horst Schmidt Kliniken Wiesbaden-N.

Frickhofen, B. Jung.

Rems-Murr-Kliniken Winnenden-S. Parmentier.

Klinikum Wolfsburg-S. Neumann.

Heinrich-Braun-Klinikum Zwickau-S. Graupner.

Supplementary Information The online version contains supplementary material available at https://doi.org/10.1007/s00432-020-03139-4.
Acknowledgements Open Access funding provided by Projekt DEAL. Sebastian Birndt was supported by the Jena Interdisciplinary Centre of Clinical Research (IZKF). The HLH-registry was funded by Grant 2014 KN 0024 of the Thüringer Aufbaubank.

\section{Compliance with ethical standards}

Conflict of interest The authors declare no conflicts of interest.

Ethical approval This study was approved by the ethics committee of the Friedrich-Schiller University Jena (3728-03/13) and carried out in accordance with the 1964 Declaration of Helsinki and its later amendments. Written informed consent was obtained from patients before enrollment, genetic analysis, and data entry.

Open Access This article is licensed under a Creative Commons Attribution 4.0 International License, which permits use, sharing, adaptation, distribution and reproduction in any medium or format, as long as you give appropriate credit to the original author(s) and the source, provide a link to the Creative Commons licence, and indicate if changes were made. The images or other third party material in this article are included in the article's Creative Commons licence, unless indicated otherwise in a credit line to the material. If material is not included in the article's Creative Commons licence and your intended use is not permitted by statutory regulation or exceeds the permitted use, you will need to obtain permission directly from the copyright holder. To view a copy of this licence, visit http://creativecommons.org/licenses/by/4.0/.

\section{References}

Allen CE, Yu X, Kozinetz CA, McClain KL (2008) Highly elevated ferritin levels and the diagnosis of hemophagocytic lymphohistiocytosis. Pediatric Blood Cancer 50:1227-1235. https://doi.org/ $10.1002 / p b c .21423$

Arca M et al (2015) Prognostic factors of early death in a cohort of 162 adult haemophagocytic syndrome: impact of triggering disease and early treatment with etoposide. Br J Haematol 168:63-68. https://doi.org/10.1111/bjh.13102

Bachier Rodriguez L, Ritchie EK (2016) A case series of adult secondary hemophagocytic lymphohistiocytosis treated at weill cornell medical college. Blood 128:4874-4874

Bergsten E et al (2017) Confirmed efficacy of etoposide and dexamethasone in HLH treatment: long-term results of the cooperative HLH-2004 study. Blood 130:2728-2738. https://doi.org/10.1182/ blood-2017-06-788349

Berliner N, Kurra C, Chou D (2016) CASE RECORDS of the MASSACHUSETTS GENERAL HOSPITAL. Case 1-2016. An 18-yearold man with fever abdominal pain, and thrombocytopenia. $\mathrm{N}$ Engl J Med 374:165-173. https://doi.org/10.1056/NEJMcpc150 1306

Bode SF et al (2014) Hemophagocytic lymphohistiocytosis in imported pediatric visceral leishmaniasis in a nonendemic area. J Pediatr 165(147-153):e141. https://doi.org/10.1016/j.jpeds.2014.03.047

Bodley Scott R, Robb-Smith AHT (1939) Histiocytic medullary reticulosis. The Lancet 234:194-198. https://doi.org/10.1016/S01406736(00)61951-7

Brisse E, Wouters CH, Matthys P (2016) Advances in the pathogenesis of primary and secondary haemophagocytic lymphohistiocytosis: differences and similarities. Br J Haematol 174:203-217. https:// doi.org/10.1111/bjh.14147 
Busiello R, Fimiani G, Miano MG, Arico M, Santoro A, Ursini MV, Pignata C (2006) A91V perforin variation in healthy subjects and FHLH patients. Int J Immunogenet 33:123-125. https://doi.org/ 10.1111/j.1744-313X.2006.00582.x

Cetica V et al (2016) Genetic predisposition to hemophagocytic lymphohistiocytosis: report on 500 patients from the Italian registry. J Allergy Clin Immunol 137:188-196 e184. https://doi.org/10. 1016/j.jaci.2015.06.048

Daver $\mathrm{N}$ et al (2017) A consensus review on malignancy-associated hemophagocytic lymphohistiocytosis in adults. Cancer 123:32293240. https://doi.org/10.1002/cncr.30826

Delavigne K et al (2014) Hemophagocytic syndrome in patients with acute myeloid leukemia undergoing intensive chemotherapy. Haematologica 99:474-480. https://doi.org/10.3324/haematol.2013. 097394

Ehl S et al (2018) Recommendations for the use of etoposide-based therapy and bone marrow transplantation for the treatment of HLH: consensus statements by the HLH steering committee of the histiocyte society. J Allergy Clin Immunol Pract 6:1508-1517. https://doi.org/10.1016/j.jaip.2018.05.031

Emile JF et al (2016) Revised classification of histiocytoses and neoplasms of the macrophage-dendritic cell lineages. Blood 127:2672-2681. https://doi.org/10.1182/blood-2016-01-690636

Fardet L et al (2010) Reactive haemophagocytic syndrome in 58 HIV1-infected patients: clinical features, underlying diseases and prognosis Aids 24:1299-1306. https://doi.org/10.1097/QAD. 0b013e328339e 55b

Fardet L et al (2014) Development and validation of the HScore, a score for the diagnosis of reactive hemophagocytic syndrome. Arthritis Rheumatol 66:2613-2620. https://doi.org/10.1002/art. 38690

Frimmel S, Schipper J, Henschel J, Yu TT, Mitzner SR, Koball S (2014) First description of single-pass albumin dialysis combined with cytokine adsorption in fulminant liver failure and hemophagocytic syndrome resulting from generalized herpes simplex virus 1 infection. Liver Transpl 20:1523-1524. https:// doi.org/10.1002/lt.24005

Gars E, Purington N, Scott G, Chisholm K, Gratzinger D, Martin BA, Ohgami RS (2018) Bone marrow histomorphological criteria can accurately diagnose hemophagocytic lymphohistiocytosis. Haematologica 103:1635-1641. https://doi.org/10.3324/haema tol.2017.186627

Greil C, Roether F, La Rosee P, Grimbacher B, Duerschmied D, Warnatz K (2017) Rescue of cytokine storm due to hlh by hemoadsorption in a CTLA4-deficient patient. J Clin Immunol 37:273-276

Gupta A, Weitzman S, Abdelhaleem M (2008) The role of hemophagocytosis in bone marrow aspirates in the diagnosis of hemophagocytic lymphohistiocytosis. Pediatr Blood Cancer 50:192-194. https://doi.org/10.1002/pbc.21441

Halacli B et al (2016) Investigation of hemophagocytic lymphohistiocytosis in severe sepsis patients. J Crit Care 35:185-190. https:// doi.org/10.1016/j.jcrc.2016.04.034

Han AR et al (2007) Lymphoma-associated hemophagocytic syndrome: clinical features and treatment outcome. Ann Hematol 86:493-498. https://doi.org/10.1007/s00277-007-0278-6

Hayden A et al (2017) Soluble interleukin-2 receptor is a sensitive diagnostic test in adult HLH. Blood Adv 1:2529-2534. https:// doi.org/10.1182/bloodadvances.2017012310

Hayden A, Park S, Giustini D, Lee AY, Chen LY (2016) Hemophagocytic syndromes (HPSs) including hemophagocytic lymphohistiocytosis (HLH) in adults: a systematic scoping review. Blood Rev 30:411-420 https://doi.org/10.1016/j.blre.2016.05.001

Henkes M, Finke J, Warnatz K, Ammann S, Stadt UZ, Janka G, Brugger W (2015) Late-onset hemophagocytic lymphohistiocytosis (HLH) in an adult female with Griscelli syndrome type
2 (GS2). Ann Hematol 94:1057-1060. https://doi.org/10.1007/ s00277-014-2284-9

Henter JI et al (2007) HLH-2004: Diagnostic and therapeutic guidelines for hemophagocytic lymphohistiocytosis. Pediatr Blood Cancer 48:124-131. https://doi.org/10.1002/pbc.21039

Janka GE, Lehmberg K (2014) Hemophagocytic syndromes-an update Blood Rev 28:135-142. https://doi.org/10.1016/j.blre. 2014.03.002

Jing-Shi W, Yi-Ni W, Lin W, Zhao W (2015) Splenectomy as a treatment for adults with relapsed hemophagocytic lymphohistiocytosis of unknown cause. Ann Hematol 94:753-760. https://doi.org/ 10.1007/s00277-014-2276-9

Jordan $\mathrm{M}$ et al (2015) A novel targeted approach to the treatment of hemophagocytic lymphohistiocytosis (HLH) with an anti-interferon gamma (IFN $\gamma$ ) monoclonal antibody (mAb), NI-0501: first results from a Pilot phase 2 study in children with primary HLH. Blood 126:LBA-3-LBA-3

La Rosee P (2015a) First prospective clinical trial in adult HLH. Blood 126:2169-2171. https://doi.org/10.1182/blood-2015-09-666503

La Rosee P (2015b) Treatment of hemophagocytic lymphohistiocytosis in adults. Hematol Am Soc Hematol Educ Prog 2015:190-196. https://doi.org/10.1182/asheducation-2015.1.190

La Rosee P (2016) Alleviating the storm: ruxolitinib in HLH. Blood 127:1626-1627. https://doi.org/10.1182/blood-2016-02-697151

Lachmann G, Spies C, Schenk T, Brunkhorst FM, Balzer F, La Rosee P (2018) Hemophagocytic lymphohistiocytosis: potentially underdiagnosed in intensive care units. Shock 50:149-155. https://doi. org/10.1097/SHK.0000000000001048

Lee DW et al (2014) Current concepts in the diagnosis and management of cytokine release syndrome. Blood 124:188-195. https:// doi.org/10.1182/blood-2014-05-552729

Lehmberg K, Ehl S (2013) Diagnostic evaluation of patients with suspected haemophagocytic lymphohistiocytosis. Br J Haematol 160:275-287. https://doi.org/10.1111/bjh.12138

Lehmberg K et al (2015) Consensus recommendations for the diagnosis and management of hemophagocytic lymphohistiocytosis associated with malignancies. Haematologica 100:997-1004. https:// doi.org/10.3324/haematol.2015.123562

Li J, Wang Q, Zheng W, Ma J, Zhang W, Wang W, Tian X (2014) Hemophagocytic lymphohistiocytosis: clinical analysis of 103 adult patients Medicine 93:100-105. https://doi.org/10.1097/MD. 0000000000000022

Li F et al (2015) Clinical characteristics and prognostic factors of adult hemophagocytic syndrome patients: a retrospective study of increasing awareness of a disease from a single-center in China. Orphanet J Rare Dis 10:20. https://doi.org/10.1186/ s13023-015-0224-y

Lin $M$ et al (2017) Clinical utility of soluble interleukin-2 receptor in hemophagocytic syndromes: a systematic scoping review. Ann Hematol 96:1241-1251. https://doi.org/10.1007/ s00277-017-2993-y

Machaczka M, Vaktnas J, Klimkowska M, Hagglund H (2011) Malignancy-associated hemophagocytic lymphohistiocytosis in adults: a retrospective population-based analysis from a single center. Leuk Lymphoma 52:613-619. https://doi.org/10.3109/10428194. 2010.551153

Oto M, Yoshitsugu K, Uneda S, Nagamine M, Yoshida M (2015) Prognostic factors and outcomes of adult-onset hemophagocytic lymphohistiocytosis: a retrospective analysis of 34 cases. Hematol Rep 7:5841. https://doi.org/10.4081/hr.2015.5841

Otrock ZK, Eby CS (2015) Clinical characteristics, prognostic factors, and outcomes of adult patients with hemophagocytic lymphohistiocytosis. Am J Hematol 90:220-224 https://doi.org/10.1002/ ajh. 23911

Otrock ZK, Hock KG, Riley SB, de Witte T, Eby CS, Scott MG (2017) Elevated serum ferritin is not specific for hemophagocytic 
lymphohistiocytosis. Ann Hematol. https://doi.org/10.1007/ s00277-017-3072-0

Parikh SA, Kapoor P, Letendre L, Kumar S, Wolanskyj AP (2014) Prognostic factors and outcomes of adults with hemophagocytic lymphohistiocytosis. Mayo Clin Proc 89:484-492. https://doi.org/ 10.1016/j.mayocp.2013.12.012

Park HS et al (2012) Clinical features of adult patients with secondary hemophagocytic lymphohistiocytosis from causes other than lymphoma: an analysis of treatment outcome and prognostic factors. Ann Hematol 91:897-904. https://doi.org/10.1007/ s00277-011-1380-3

Ramos-Casals M, Brito-Zeron P, Lopez-Guillermo A, Khamashta MA, Bosch X (2014) Adult haemophagocytic syndrome. Lancet 383:1503-1516. https://doi.org/10.1016/S0140-6736(13)61048-X

Riviere S, Galicier L, Coppo P, Marzac C, Aumont C, Lambotte O, Fardet L (2014) Reactive hemophagocytic syndrome in adults: a retrospective analysis of 162 patients. Am J Med 127:1118-1125. https://doi.org/10.1016/j.amjmed.2014.04.034

Schram AM et al (2016) Haemophagocytic lymphohistiocytosis in adults: a multicentre case series over 7 years. Br J Haematol 172:412-419. https://doi.org/10.1111/bjh.13837

Schram AM, Campigotto F, Mullally A, Fogerty A, Massarotti E, Neuberg D, Berliner N (2015) Marked hyperferritinemia does not predict for HLH in the adult population. Blood 125:1548-1552. https://doi.org/10.1182/blood-2014-10-602607

Sepulveda FE, de Saint BG (2017) Hemophagocytic syndrome: primary forms and predisposing conditions. Curr Opin Immunol 49:20-26. https://doi.org/10.1016/j.coi.2017.08.004

Shabbir M, Lucas J, Lazarchick J, Shirai K (2011) Secondary hemophagocytic syndrome in adults: a case series of 18 patients in a single institution and a review of literature. Hematol Oncol 29:100-106 https://doi.org/10.1002/hon.960

Sin JH, Zangardi ML (2017) Ruxolitinib for secondary hemophagocytic lymphohistiocytosis: first case report. Hematol Oncol Stem Cell Ther. https://doi.org/10.1016/j.hemonc.2017.07.002
Tamamyan GN et al (2016) Malignancy-associated hemophagocytic lymphohistiocytosis in adults: relation to hemophagocytosis, characteristics, and outcomes. Cancer 122:2857-2866. https://doi.org/ $10.1002 /$ cncr.30084

Teachey DT et al (2013) Cytokine release syndrome after blinatumomab treatment related to abnormal macrophage activation and ameliorated with cytokine-directed therapy. Blood 121:51545157. https://doi.org/10.1182/blood-2013-02-485623

Trottestam H et al (2011) Chemoimmunotherapy for hemophagocytic lymphohistiocytosis: long-term results of the HLH-94 treatment protocol. Blood 118:4577-4584 https://doi.org/10.1182/ blood-2011-06-356261

Tschistowitsch T, Bykowa O (1928) Retikulose als eine Systemerkrankung der blutbildenden. Organe Virchows Arch A 267:91105. https://doi.org/10.1007/bf02029855

Tsuji T, Hirano T, Yamasaki H, Tsuji M, Tsuda H (2014) A high sIL$2 \mathrm{R} /$ ferritin ratio is a useful marker for the diagnosis of lymphomaassociated hemophagocytic syndrome. Ann Hematol 93:821-826. https://doi.org/10.1007/s00277-013-1925-8

Wang Y et al (2014) Genetic features of late onset primary hemophagocytic lymphohistiocytosis in adolescence or adulthood. PLoS ONE 9:e107386. https://doi.org/10.1371/journal.pone.0107386

Zhang K et al (2011a) Hypomorphic mutations in PRF1, MUNC13-4, and STXBP2 are associated with adult-onset familial HLH. Blood 118:5794-5798. https://doi.org/10.1182/blood-2011-07-370148

Zur Stadt U, Beutel K, Weber B, Kabisch H, Schneppenheim R, Janka $\mathrm{G}$ (2004) A91V is a polymorphism in the perforin gene not causative of an FHLH phenotype. Blood 104:1909. https://doi.org/10. 1182/blood-2004-02-0733. (Author reply 1910)

Publisher's Note Springer Nature remains neutral with regard to jurisdictional claims in published maps and institutional affiliations.

\section{Authors and Affiliations}

\section{Sebastian Birndt ${ }^{1}(1)$. Thomas Schenk ${ }^{1} \cdot$ Babett Heinevetter $^{1} \cdot$ Frank M. Brunkhorst ${ }^{2} \cdot$ Georg Maschmeyer $^{3}$. Frank Rothmann ${ }^{3}$. Thomas Weber ${ }^{4} \cdot$ Markus Müller $^{5}$. Jens Panse ${ }^{6}$. Olaf Penack ${ }^{7}$. Roland Schroers ${ }^{8}$. Jan Braess ${ }^{9}$. Norbert Frickhofen ${ }^{10}$. Stephan Ehl ${ }^{11}$. Gritta Janka ${ }^{12} \cdot$ Kai Lehmberg $^{12} \cdot$ Mathias W. Pletz $^{13} \cdot$ Andreas Hochhaus $^{1}$. Thomas Ernst ${ }^{1} \cdot$ Paul La Rosée ${ }^{14}$}

1 Klinik für Innere Medizin II, Abt. Hämatologie und Intern. Onkologie, Universitätsklinikum Jena, Jena, Germany

2 Zentrum für Klinische Studien, Universitätsklinikum Jena, Jena, Germany

3 Klinik für Hämatologie, Onkologie u. Palliativmedizin, Klinikum Ernst Von Bergmann, Potsdam, Germany

4 Klinik für Hämatologie und Onkologie, Universitätsklinikum Halle (Saale), Halle, Germany

5 Zentrum für Infektiologie und HIV, Vivantes Auguste-Viktoria-Klinikum, Berlin, Germany

6 Klinik für Hämatologie, Onkologie, Hämostaseologie und Stammzelltransplantation, Uniklinik RWTH Aachen, Aachen, Germany

7 Medizinische Klinik mit Schwerpunkt Hämatologie, Onkologie und Tumorimmunologie, Charité Universitätsmedizin, Berlin, Germany
8 Hämatologie und Onkologie, Universitätsklinikum Knappschaftskrankenhaus, Bochum, Germany

9 Onkologie und Hämatologie, Krankenhaus Barmherzige Brüder, Regensburg, Germany

10 Hämatologie, Onkologie und Palliativmedizin, HELIOS Dr. Horst Schmidt Kliniken, Wiesbaden, Germany

11 Centrum für Chronische Immundefizienz (CCI), Universitätsklinikum Freiburg, Freiburg, Germany

12 Pädiatrische Hämatologie und Onkologie, Universitätsklinikum Eppendorf, Hamburg, Germany

13 Institut für Infektionsmedizin und Krankenhaushygiene, Universitätsklinikum Jena, Jena, Germany

14 Klinik für Innere Medizin II, Hämatologie, Onkologie, Immunologie, Infektiologie und Palliativmedizin, Schwarzwald-Baar Klinikum, Klinikstr. 11, 78052 Villingen-Schwenningen, Germany 